\title{
Evaluation of the psychometric properties of the Early Morning Symptoms of COPD Instrument (EMSCl)
}

This article was published in the following Dove Press journal: International Journal of COPD

\author{
Asha Hareendran' \\ Barry J Make ${ }^{2}$ \\ Erica Zaiser ${ }^{1}$ \\ Esther Garcia Gil ${ }^{3}$ \\ 'Patient Centered Research, Evidera, \\ London, UK; ${ }^{2}$ Division of Pulmonary, \\ Critical Care and Sleep Medicine, \\ National Jewish Health, Denver, \\ CO, USA; ${ }^{3}$ Global Medical Affairs, \\ AstraZeneca, Barcelona, Spain
}

Background: Early morning respiratory symptoms impact quality of life and are often the most troublesome for patients with COPD. Reduction in symptoms and their impact are important treatment outcomes for COPD. The Early Morning Symptoms of COPD Instrument (EMSCI) is a daily diary designed to collect patients' report of the occurrence, severity, and impact of morning COPD symptoms.

Methods: To assess the psychometric properties of the EMSCI, a split-half sample of data from a COPD clinical trial where participants completed the EMSCI daily was used for conducting descriptive statistics, factor analyses, and Rasch model analyses to examine item performance and inform scoring. Once the final scoring algorithm was determined, data from the second split-half sample were used to examine the properties of the EMSCI. Test-retest reliability was assessed using intraclass correlation coefficient (ICC). Correlations with other study assessments were used to evaluate convergent and known-groups validity.

Results: Data from 1,663 patients with COPD aged 40-93 years were analyzed. Factor analysis and Rasch analysis confirmed a one-factor structure for the 6 individual symptom items. Item analyses supported the generation of 4 scores. All scores demonstrated good test-retest reliability: 6-item symptom severity (ICC, 0.84); overall morning symptom severity (ICC, 0.84); activity limitation (ICC, 0.85); and rescue medication (ICC, 0.62). Significant correlations between EMSCI scores, St George's Respiratory Questionnaire scores, and EXAcerbations of Chronic pulmonary disease Tool (EXACT)-Respiratory Symptoms scores supported the tool's convergent validity. Significant differences $(p<0.0001)$ in all EMSCI domain scores were found between known-groups based on median split St George's Respiratory Questionnaire and EXACT-Respiratory Symptoms scores.

Conclusion: The EMSCI consists of 4 scores: 6-item symptom severity, overall symptom severity, activity limitation, and rescue medication. The EMSCI is a reliable and valid instrument for evaluating patients' experience of early morning COPD symptoms.

Keywords: respiratory symptoms, daily diary, PRO, psychometric validation, activity limitation, rescue medication

\section{Introduction}

COPD is a chronic lung condition characterized by airflow obstruction. ${ }^{1}$ Common symptoms include shortness of breath, cough, wheeze, and phlegm production, but symptoms can vary across individuals. Patients with COPD often report the morning as a particularly difficult time ${ }^{2}$ when symptoms can limit their ability to perform morning activities ${ }^{3,4}$ and negatively impact their quality of life (QOL). ${ }^{5}$ A pan-European, cross-sectional, observational study conducted in 17 countries found early morning
Correspondence: Asha Hareendran Evidera, Metro Building 6th Floor, I Butterwick, London W6 8DL, UK Tel +442085765000

Fax +442085765I95

Email asha.hareendran@evidera.com 
symptoms $(\mathrm{EMS})^{3}$ were the worst symptoms experienced in patients with COPD. ${ }^{3}$ EMS can also potentially predict worsening of the disease, increasing the risk for exacerbations and use of rescue inhalers. ${ }^{6}$ Reducing EMS and their impact on patients' QOL is a key goal of treatment, and there is a need to assess EMS to evaluate the necessity and benefits of treatments.

Disease symptoms and their impact on patients are best measured using patient-reported outcome (PRO) measures, which are developed using methods that ensure the measure reflects the patient's perspective; ensuring content validity. ${ }^{7}$

A review of the literature at the time of the development of the Early Morning Symptoms of COPD Instrument (EMSCI; 2011) revealed only 2 measures reporting EMS: the Capacity of Daily Living during the Morning questionnaire $(\mathrm{CDLM})^{8}$ and Global Chest Symptoms Questionnaire (GCSQ). ${ }^{8}$ However, the symptoms measured in the GCSQ include only shortness of breath and tightness in the chest, and the development of the instruments involved only a small sample of individuals. Furthermore, there was no evidence of the saturation of concepts to support the content validity of the tools, that is, to ensure that the instrument evaluated all the concepts that were relevant to patients' experience of COPD symptoms in the early morning.

A more recent review revealed that other measures of EMS are being developed in parallel to the development of the EMSCI, for example the COPD morning symptom diary (COPD-MSD). ${ }^{9}$ The 19 -item measure is a daily diary where patients are asked about "this morning"; the diary includes items on frequency and severity of COPD symptoms in the morning, wheezing the previous night, level of shortness of breath related to specific activities in the morning, and feeling tired and rested. The 10-item Manchester Early Morning Symptoms Index (MEMSI) ${ }^{10}$ was developed based on focus groups and cognitive debriefing and has been validated among Global initiative on Obstructive Lung Disease (GOLD) grade II patients with COPD. The measure uses a 1-week recall period to evaluate EMS and impaired activity in patients with COPD.

The EMSCI ${ }^{11}$ was designed as a daily diary to collect data on the occurrence and severity of EMS and the impact of the symptoms on patients with COPD. The EMSCI was developed according to the US Food and Drug Administration Guidance for Industry on PRO measures to support labeling claims ${ }^{12}$ and developed based on a literature review of existing COPD instruments, interviews with COPD clinical experts, and qualitative research involving patients with COPD. ${ }^{11}$ The qualitative research was conducted to the point of saturation to ensure the content validity of the instrument. ${ }^{11}$
Patient involvement in the development of the instrument, a clear rationale for item generation and reduction, and strong measurement properties (reliability and validity) are necessary to ensure that an instrument captures data that are both meaningful and relevant to the target sample. ${ }^{13-15}$

\section{Objective}

The objective of this study was to conduct item analyses to inform the deletion or retention of items, determine the scoring algorithm, and examine the cross-sectional psychometric properties (reliability and validity) of the EMSCI.

\section{Methods \\ Study design}

The data used for psychometric evaluation in this study were from a prospective Phase III, multicenter, multinational, randomized, parallel-group, active and placebo-controlled clinical trial of a bronchodilator (NCT01437397) ${ }^{16}$ with a $24-w e e k$ treatment period. The original clinical trial protocol was approved by the institutional review board at each study center (Supplementary material), and all participants provided written informed consent.

\section{Study sample}

The trial randomized 1,692 participants aged $\geq 40$ years with moderate-to-severe stable COPD (defined using the criteria of the GOLD [2011]: post-albuterol/salbutamol forced expiratory volume in 1 second $\left[\mathrm{FEV}_{1}\right] \geq 30$ to $<80 \%$ predicted and $\mathrm{FEV}_{1}$ /forced vital capacity $[\mathrm{FVC}]$ of $<0.7$ ). Patients had a smoking history of 10 pack-years or more.

\section{Measures}

During the trial, patients completed a daily electronic diary that recorded the EMSCI questions and the Evaluating Respiratory Symptoms (E-RS). ${ }^{17}$ Patients were also asked to complete the St George's Respiratory Questionnaire (SGRQ) ${ }^{18}$ and the Patient Global Impression of Change (PGIC), ${ }^{19}$ and spirometry tests at study visits. Data from the E-RS and SGRQ were used to evaluate convergent validity and known-groups' validity.

\section{$\mathrm{EMSCl}$}

The EMSCI was designed to measure the occurrence and severity of EMS in patients with COPD, and the symptoms' impact on activity limitation and rescue medication use. ${ }^{11}$ Patients completed the EMSCI each morning, between 7 and 11 am, using an electronic daily diary. The EMSCI asks patients to indicate symptoms between the time they got out 
of bed to start their day and the time of diary completion. The EMSCI asks about the presence of specific COPD symptoms (cough, wheezing, shortness of breath, tightness in chest, chest congestion, and difficulty bringing up phlegm) and their severity on a scale from 1 to 4 ( $1=$ mild, $4=$ very severe $)$. A score of 0 is assigned if a patient had no symptoms. Single items assess overall COPD symptom severity in the early morning $(0=$ no symptoms, $4=$ very severe $)$, early morning activity limitation due to COPD symptoms $(0=$ not at all, $4=\mathrm{a}$ very great deal), and the number of puffs of rescue medication required in the morning.

\section{SGRQ}

The $\mathrm{SGRQ}^{18}$ is a validated measure of impaired health in diseases of chronic airflow limitation, and has been widely used in clinical trials in COPD. It contains 50 items divided into 3 subscales: "symptoms", "activity", and "impacts". A score is calculated for each section and a "total" score is also calculated. In each case, the lowest possible value is zero and the highest is 100 . Higher values correspond to greater impairment of QOL.

\section{E-RS}

The E-RS was designed as a standardized respiratory symptom diary and utilizes 11 respiratory symptom items from the 14-item Exacerbations of Chronic Pulmonary Disease Tool. ${ }^{17}$ The respiratory symptoms total score represents overall respiratory symptom severity. Total scores range from 0 to 40 , with higher scores indicating more severe respiratory symptoms. The E-RS was completed by patients throughout the course of the study in the e-Diary each night directly before going to sleep.

\section{PGIC}

The $\mathrm{PGIC}^{19}$ contains an item that assesses the patient's perspective on how their COPD symptoms have changed since the start of the study. The PGIC has a 7-point response grade ranging from "very much worse" to "very much improved" (scores $1-3=$ improved, $4=$ no change, and 5-7= worse). The PGIC provides a subjective summary index of degree of improvement (or worsening). The PGIC was used to identify a stable sample of patients for evaluating the EMSCI's test-retest validity.

\section{Spirometry}

Spirometry $\left(\mathrm{FEV}_{1}\right.$ and $\left.\mathrm{FVC}\right)$ was performed by clinical study site personnel during study visits. $\mathrm{FEV}_{1}$ measures how much air a person can exhale during a forced breath, and FVC is the total amount of air exhaled during the $\mathrm{FEV}_{1}$ test. The largest value of 3 technically satisfactory forced exhalation efforts was used in the current study.

\section{Statistical analyses}

Statistical analyses were conducted in 2 stages and in accordance with best practices for instrument development and psychometric evaluation. ${ }^{14}$ The analytic sample consisted of patients in the intention-to-treat population $(n=1,669)$ with a baseline EMSCI measurement. Individuals were excluded if data were missing on PRO and clinical data at baseline, leaving a sample of 1,663 patients. The data for all treatment groups were combined and randomly split into 2 split-half samples for the analyses. Phase I consisted of item analyses and the factor analysis to inform item reduction and for defining scoring. Item analyses, factor analysis, and scoring definition utilized the first split-half sample $(\mathrm{n}=831)$. Using the final version of the EMSCI and scoring algorithm, Phase II was conducted on the second split-half sample $(\mathrm{n}=832)$ to determine reliability and validity. Statistical analyses were performed using SAS statistical software version 9.1 (Cary, NC, USA) following a pre-specified statistical analysis plan. Item analysis and psychometric validation were conducted using the average of the 7 days (Day -6 to Day 1) leading up to and including the day of randomization (referred to in this manuscript as baseline week), and a single day score; Day -7 before randomization (referred to as baseline day). For analysis of a 7-day average, a minimum of 4 (of 7) diary entries during the 7-day period was required, following the approach of Junghard et al. ${ }^{20}$ Tests of validity (construct and known-groups [discriminant] validity) were performed on samples at baseline week and Week 4/Visit 4 (the average of the week before Visit 4 or Day 29 \pm 3 ).

\section{Item structure and scoring algorithm development}

Item analysis was conducted to determine if any items were poorly performing and to determine the scoring algorithm for the measure. Distributional characteristics of each of the 9 items on the EMSCI were evaluated by exploring item-level descriptive statistics and item-to-item correlations.

Criteria for identifying poorly fitting items included any items that showed a floor (minimum response $>30 \%$ of patients) or ceiling (maximum response $>30 \%$ of patients) effect; item-item correlations $>0.80$; factor loadings $<0.3$ or misfit to the Rasch model; a high negative $(<-3.0)$ residual number, which suggests an over-fitting item (meaning that the information provided by this item does not add any new information to the measurement); or a high positive $(>3.0)$ 
residual number, which suggests that the item is underfitting (indicating that the item has a poor fit to the model and the response categories are under-discriminating-or not discriminating-differences in severity). To evaluate the model fit, the following thresholds were used: comparative fit index $\geq 0.90$, root mean square error of approximately $\leq 0.08$, and root mean square residual number $\leq 0.05$. The results from the confirmatory factor analysis (CFA) and Rasch model analysis were used to inform item deletion or retention of items through an iterative process and to determine the scoring algorithm for the measure.

After the initial item evaluation, factor analysis, and Rasch model analysis, an item analyses meeting was conducted with the PRO tool developers, statisticians, and a COPD clinical expert (co-authors of this paper, including $\mathrm{BM}, \mathrm{AH}$, and EZ). The intent of the meeting was to consider the qualitative information obtained during the concept elicitation and cognitive interviews, ${ }^{11}$ and incorporate the quantitative results to develop a scoring scheme that is consistent with the objectives of the measure. Clinical input and item performance results were used to identify well-performing items as well as items that were potentially problematic. All decisions made on the final items were with the qualitative results of patient input in mind and the consideration of clinically relevant symptoms as well as the quantitative results on item performance.

Internal consistency (reliability), test-retest reliability, construct validity, and known-groups (discriminant) validity were all examined as part of the psychometric assessment of the EMSCI.

Internal consistency was assessed using Cronbach's $\alpha$, with a target value $>0.70$ at baseline week. Test-retest reliability was assessed using intraclass correlation coefficient (ICC) within a group of stable patients; defined by PGIC "no change" (n=222). Patients' scores were compared at baseline week and Visit 4 (average of week ending on study Visit 4). An ICC $\geq 0.7$ indicates good test-retest reliability, between 0.4 and 0.7 indicates moderate reliability, and $<0.4$ indicates low test-retest reliability. ${ }^{21,22}$

Construct validity (ie, the extent to which a scale measures what it is hypothesized to measure) was evaluated at baseline by examining the relationship between each domain score with SGRQ total and subscale scores, E-RS total score, and $\mathrm{FEV}_{1}$. Construct validity was considered supported when the EMSCI domain items/scores were substantially correlated ( $>0.40$; using Spearman rank order correlation coefficients) with items or scales measuring similar concepts. ${ }^{23}$
Known-groups validity was evaluated using analysis of variance models to explore the relationship between the EMSCI scores and measures of disease status: severity of airflow obstruction from GOLD stage I (mild) to stage IV (very severe), SGRQ total score $(\leq$ sample median versus $>$ sample median), SGRQ symptoms score ( $\leq$ sample median versus $>$ sample median), and E-RS total score $(\leq$ sample median versus $>$ sample median). EMSCI scores were compared between groups differing on severity of their airflow obstruction (both were collected during screening), SGRQ scores (at baseline), and E-RS total score (at baseline).

\section{Results}

The demographic and clinical characteristics of the full sample $(n=1,663)$ are presented in Table 1 . The mean age was $63.9 \pm 8.9$ years (range: $40-93$ years old) and the majority of participants $(\mathrm{n}=885 ; 53.2 \%)$ were male. As expected, given the inclusion criteria, most participants were categorized as GOLD stage II $(n=946 ; 56.9 \%)$ or III $(n=696 ; 41.9 \%)$, with only 4 participants classified as GOLD stage I (0.24\%) and 12 as GOLD stage IV $(0.72 \%)$. The most common

Table I Sociodemographic and clinical characteristics

\begin{tabular}{|c|c|}
\hline \multirow[t]{2}{*}{ Characteristics } & \multirow{2}{*}{$\begin{array}{l}\text { Overall sample } \\
\text { Day } I,{ }^{a} N=1,663 \\
\end{array}$} \\
\hline & \\
\hline \multicolumn{2}{|l|}{ Demographic } \\
\hline Age, mean (SD) & $63.9(8.9)$ \\
\hline Gender, female, n (\%) & $778(46.8)$ \\
\hline \multicolumn{2}{|l|}{ Race/ethnicity, \% } \\
\hline White & 93.2 \\
\hline Black or African American & 5.7 \\
\hline Other & I.I \\
\hline $\mathrm{FEV}_{\text {, }}$ mean $(\mathrm{SD})$ & $\mathrm{I} .4(0.5)$ \\
\hline $\mathrm{FEV}_{1} \%$ predicted mean (SD) & $49(14.0)$ \\
\hline \multicolumn{2}{|l|}{$\operatorname{SGRQ}^{b}(n=I, 648)$} \\
\hline Total score mean (SD) & $46.5(18.5)$ \\
\hline Symptoms domain score mean (SD) & $65.1(21.4)$ \\
\hline Activity domain score mean (SD) & $60.6(22.3)$ \\
\hline Impact domain score mean (SD) & $32.7(19.4)$ \\
\hline E-RS $(n=1,658)$ total $^{c}$ mean $(S D)$ & $9.6(6.3)$ \\
\hline \multicolumn{2}{|l|}{ GOLD stage, $\mathrm{n}(\%)$} \\
\hline I & $4(0.2)$ \\
\hline ॥ & $946(56.9)$ \\
\hline III & $696(41.9)$ \\
\hline IV & $12(0.7)$ \\
\hline BDI mean (SD) & $6.4(2.2)$ \\
\hline
\end{tabular}

Notes: ${ }^{\text {FFor }}$ patients who were missing the EMSCl diary entry on Day I (day of randomization), data from the closest day before Day I were used. 'SGRQ scores range from 0 to 100; higher score $=$ more severe health status. ${ }^{\mathrm{C}} \mathrm{T}$ he $\mathrm{E}-\mathrm{RS}$ total score is an aggregate of 3 domains identified on the E-RS. Scores range from 0 to 40; higher score $=$ more severe respiratory symptoms.

Abbreviations: BDI, Baseline Dyspnea Index; EMSCI, Early Morning Symptoms of COPD Instrument; E-RS, Evaluating Respiratory Symptoms; FEV , forced expiratory volume in I second; GOLD, Global initiative for chronic Obstructive Lung Disease; SGRQ, St George's Respiratory Questionnaire. 
COPD-related concomitant medication was a short-acting $\beta 2$-adrenergic agonist ( $n=940 ; 56.5 \%)$, followed by inhaled corticosteroids $(n=549 ; 33.0 \%)$, and systemic corticosteroids $(\mathrm{n}=243 ; 14.6 \%)$. Long-acting bronchodilators were not allowed for the duration of the clinical trial. Participants had an average total score of 9.6 on the E-RS and SGRQ total and symptom scores of 46.5 and 65.1 , respectively, suggesting moderate-to-severe COPD. ${ }^{24}$

\section{Patients' experiences with COPD symptoms in the early morning}

Within the full sample, at baseline day, more than $50 \%$ of patients experienced coughing and shortness of breath, and between $23 \%$ and $42 \%$ of patients experienced the other symptoms of COPD (Figure 1). Approximately 29\% of the full sample experienced EMS ("Overall, how severe were your COPD symptoms this morning?") that were at least moderate (score $\geq 2$ ) in severity on baseline day.

\section{Item analysis for item reduction}

The full range of response options $(0-4)$ was observed for all items on the EMSCI. The percentage showing ceiling effects was low for all items, ranging from $0.1 \%$ to $1.1 \%$. Floor effects were observed for all the 6 symptom items ( $>30 \%$ not experiencing the symptom in the time period) on the baseline day, ranging from $42.0 \%$ to $76.9 \%$. The single overall symptom severity item did not show floor effects $(17.5 \%)$. These findings suggest that patients did not experience all symptoms on any given day; however, floor effects decreased for each of the 6 symptom items when using the average at baseline week (Table 2).

Item-item correlations for the 6 symptom items and the overall symptom severity item were between 0.28 and 0.80 at baseline week and statistically significant $(p<0.0001)$. There were high correlations between the single item measuring overall symptom severity and each of the 6 individual symptom items at baseline week $(>0.51, p$-values all $<0.0001)$. In particular, the overall symptom severity item was very strongly correlated with severity of shortness of breath (0.80). The predetermined cutoff for identifying potential item redundancy was a correlation of 0.80 , a threshold reached at baseline week. Excluding the correlation between these 2 items, the correlations between EMSCI symptom items ranged from 0.28 to 0.70 (baseline week). However, the high item-to-item correlation suggests that shortness of breath may be an important cardinal symptom of COPD. The items measure different concepts; one assesses a specific symptom (shortness of breath), the other assesses the patients' overall experience of COPD symptoms in the morning, and may also include symptoms other than the 6 key COPD symptoms specifically noted in the EMSCI. Therefore a decision was made to retain both items. In addition, clinical input suggested that all individual symptom items were clinically meaningful and should be retained for a full understanding of the patient experience with COPD EMS. A summary of the item analyses for decision making can be found in Table 2 .

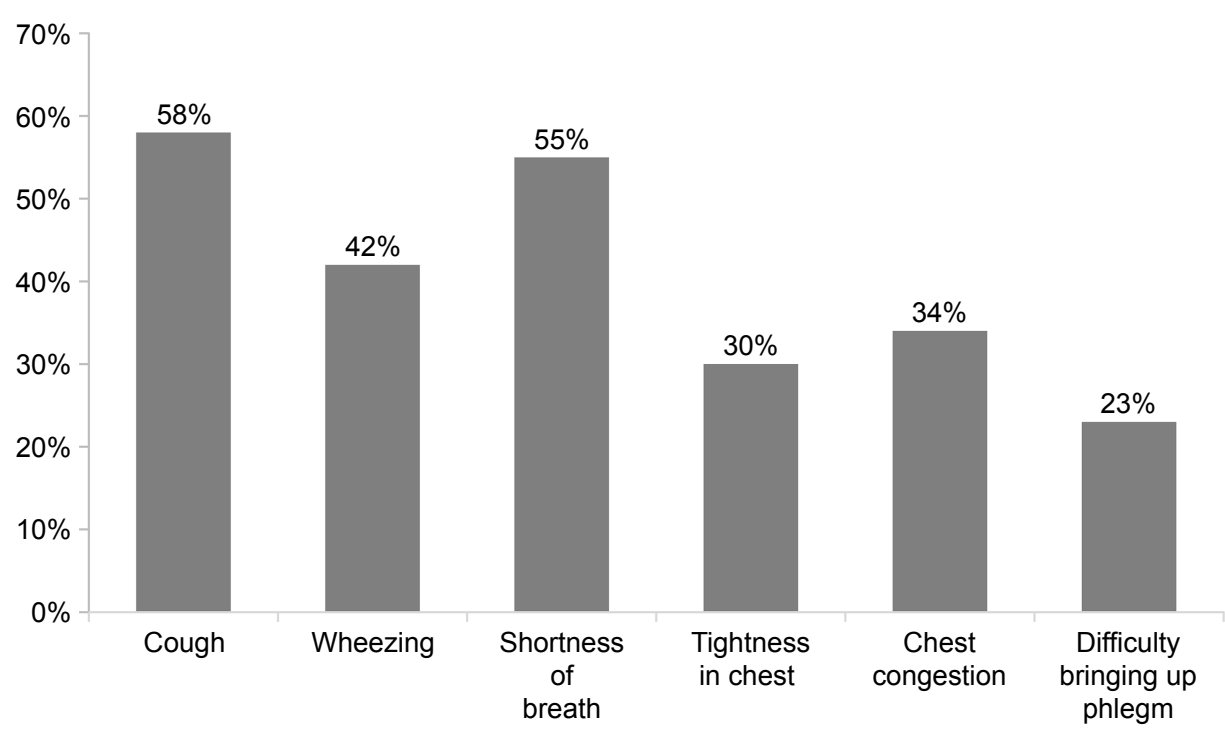

Figure I Percentage of patients experiencing EMS of COPD evaluated using the EMSCl on baseline day. ${ }^{\mathrm{a}}$ Note: ${ }^{a}$ Baseline: Day -7 prior to randomization.

Abbreviations: EMS, early morning symptoms; EMSCI, Early Morning Symptoms of COPD Instrument. 
Table 2 Summary of item analyses for decision making

\begin{tabular}{|c|c|c|c|c|c|}
\hline $\mathbf{N}=\mathbf{8 3}$ I & $\begin{array}{l}\text { Floor effect } \\
\text { (baseline } \\
\text { week) }\end{array}$ & $\begin{array}{l}\text { Highest inter- } \\
\text { item correlation } \\
\text { (baseline week) }\end{array}$ & $\begin{array}{l}\text { CFA loading } \\
\text { (baseline } \\
\text { week) }\end{array}$ & IRT fit & $\begin{array}{l}\text { Decision to } \\
\text { reject or } \\
\text { accept }\end{array}$ \\
\hline Cough & $21.0 \%$ & 0.660 & 0.736 & Yes & Accept \\
\hline Wheezing & $36.8 \%^{a}$ & 0.612 & 0.745 & Yes & Accept \\
\hline Shortness of breath & $22.2 \%$ & $0.803^{\mathrm{a}}$ & 0.757 & Yes & Accept \\
\hline Tightness in chest & $48.0 \%^{\mathrm{a}}$ & 0.582 & 0.762 & Yes & Accept \\
\hline Chest congestion & $48.3 \%^{\mathrm{a}}$ & 0.598 & 0.843 & No & Accept \\
\hline Difficulty bringing up phlegm & $59.6 \%^{a}$ & 0.577 & 0.669 & Yes & Accept \\
\hline Overall symptom severity & $6.8 \%$ & $0.803^{\mathrm{a}}$ & NA & No & Accept \\
\hline Activity limitation & $34.8 \%^{\mathrm{a}}$ & 0.697 & NA & NA & Accept \\
\hline Rescue medication & NA & 0.445 & NA & NA & Accept \\
\hline
\end{tabular}

Note: andicates they are above the threshold criteria that were set a priori to flag items for potential problems, that is, floor effect minimum response $>30 \%$ and ceiling effect maximum response $>30 \%$.

Abbreviations: CFA, confirmatory factor analysis; IRT, item response theory; NA, not applicable.

The Rasch model analysis conducted at baseline day included both the overall symptom severity item and the 6 specific symptom items. In this model, the former did not fit the model (chi-square probability $p<0.001$; fit residual: -5.29 ). A second Rasch model analysis was then conducted using just the 6 symptom items.

Most items were identified as having correctly ordered response categories, with the exception of the misfitting item on chest congestion (fit $=-3.09$ ). All other items fell within the acceptable -3.0 fit residual range. The overall model-fit chi-square was $94.81(p<0.001)$. A decision was made to retain the item on chest congestion as patient interviews and insight from clinical experts suggested that it is an important symptom.

The threshold parameters for the items on tightness in the chest and bringing up phlegm suggested response option misordering. This was likely due to a small percentage of subjects who chose the response option "mild", relative to the large percentage who indicated they did not experience the symptom at all $(15.3 \%$ versus $69.5 \%$ for tightness, and $6.5 \%$ versus $76.9 \%$ for difficulty bringing up phlegm). Given that the location of the "mild" (scored as 1) response option was in between "no symptoms" (scored as 0 ) and "moderate" (scored as 2) as intended, and because it assesses a clinically relevant symptom (as determined by qualitative interviews and clinical confirmation during the meeting), the decision was made to retain the item.

\section{Determining scoring of the EMSCI}

The standardized coefficients of the 6 symptom items on the EMSCI ranged from 0.65 to 0.791 , with good model fit. CFA suggested a 1-factor structure for the 6 symptom severity items. It was decided that the 6 symptom severity items should be scored as one domain score, separate from the single item measuring overall symptom severity, and the activity limitation and rescue medication items would each be scored separately. The revised conceptual (measurement) framework of the EMSCI reflecting these changes is shown in Figure 2.

\section{Psychometric properties}

The 4 EMSCI scores - 6-item symptom severity score, overall symptom severity score, activity limitation score, and rescue medication score - were tested for their psychometric properties: reliability and validity.

\section{Reliability}

Internal consistency was high for the 6-item symptom severity score (Cronbach's $\alpha=0.82$ ), indicating good reliability.

Among stable patients (those classified as "no change" in symptoms on the PGIC from Weeks 1 to 4; $n=222$ ), good test-retest reliability (ICC $>0.7$ ) was demonstrated for the 6-item symptom severity score (ICC 0.84 ), overall symptom severity score (ICC 0.84 ), and activity limitation score (ICC 0.85). The rescue medication score (ICC 0.62) was moderately reliable.

\section{Validity}

Convergent validity results are shown in Table 3 . The 4 EMSCI scores were correlated with scores on other PRO instruments that measure similar concepts (SGRQ and E-RS). Moderate to strong correlations were observed between both the SGRQ and E-RS, and the 6-item symptom severity (all $>0.54$ ), overall symptom severity (all $>0.52$ ), and activity limitation (all $>0.52$ ) scores. Smaller, but significant correlations were observed between the rescue medication score and both the SGRQ and E-RS scores (0.32-0.35). Correlations with $\mathrm{FEV}_{1}$ were low and mostly not significant; 


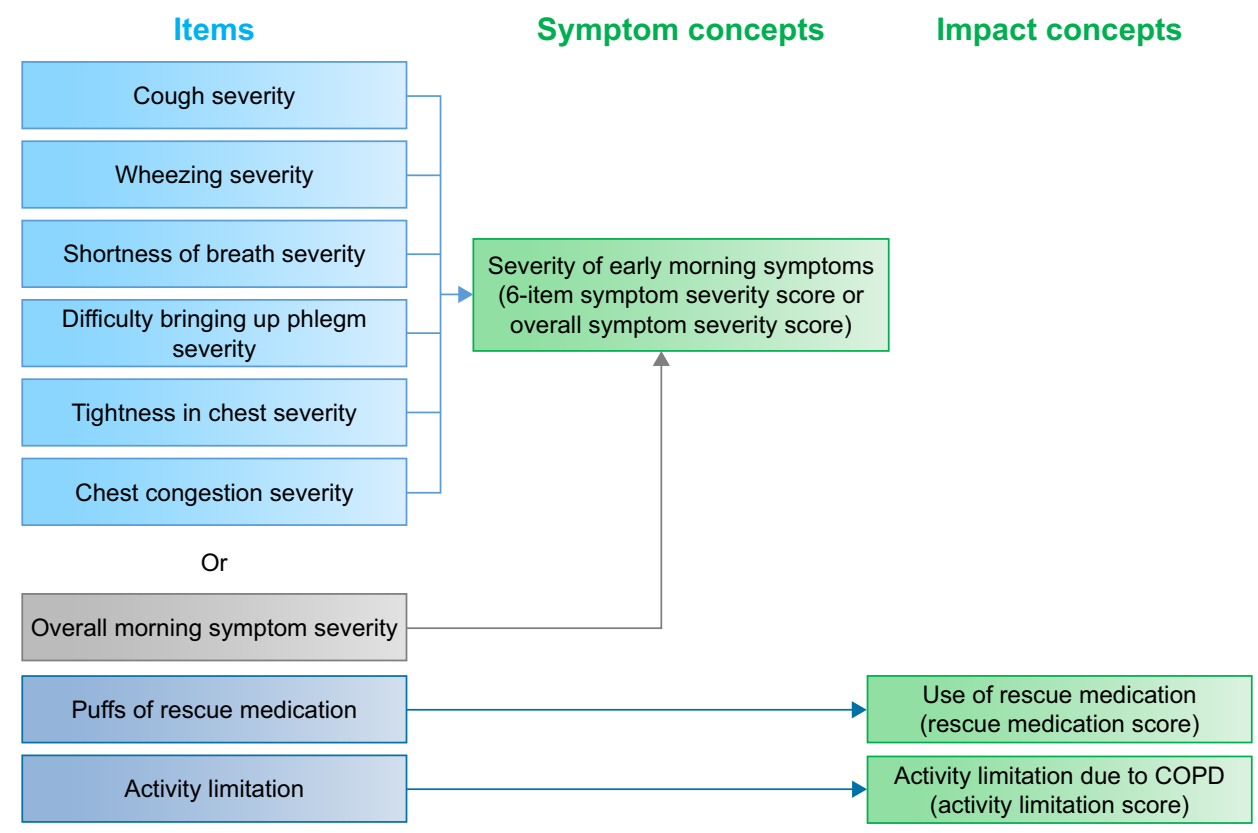

Figure 2 Final EMSCl conceptual framework.

Abbreviation: $\mathrm{EMSCl}$, Early Morning Symptoms of COPD Instrument.

this was expected as COPD symptoms have been shown to have a poor relationship with lung function parameters.

\section{Known-groups (discriminant) validity}

A pairwise comparison of EMSCI domain scores was made between GOLD stages II and III as the sample contained primarily patients with moderate and severe symptoms. Significant differences were found between these 2 groups for all EMSCI scores (Figure 3).

A comparison was made between the EMSCI domain scores and SGRQ and E-RS scores. Significant differences in all EMSCI domain scores were found between known-groups based on median-splits of SGRQ total and symptoms scores (Figure 4) and E-RS total score (Figure 5).

\section{Discussion}

EMS of COPD can significantly impact patient functioning., ${ }^{3,4}$ The EMSCI was developed based on patients' experiences of
COPD and was designed as a daily diary to measure the variability of occurrence and severity of EMS and their impact on morning activities and rescue medication use.

CFA and Rasch model analyses confirmed a 1-factor structure for the 6 symptom severity items, and following psychometric analyses and discussions with clinical experts, it was suggested that these items be scored separately to the overall symptom severity item. The final EMSCI measure is composed of 9 items and these can be used to generate 4 scores: 1 ) the 6 -item symptom severity score; 2 ) the overall symptom severity score; 3 ) the rescue medication score; and 4) the activity limitation score.

This study demonstrates that the EMSCI is a reliable and valid instrument to evaluate EMS of COPD and their impact on patients. Test-retest reliability was confirmed for all 4 scores. The 4 EMSCI scores were correlated with scores on other PRO instruments measuring similar concepts (SGRQ and E-RS) demonstrating convergent validity. In addition,

Table 3 Correlation $^{\mathrm{a}}$ of EMSCI domain scores with SGRQ, E-RS total, and FEV, ${ }_{1}^{\mathrm{b}}$ at baseline week

\begin{tabular}{lllll}
\hline Outcome measure & $\begin{array}{l}\text { 6-item symptom } \\
\text { severity }\end{array}$ & $\begin{array}{l}\text { Overall symptom } \\
\text { severity }\end{array}$ & $\begin{array}{l}\text { Activity } \\
\text { limitation }\end{array}$ & $\begin{array}{l}\text { Rescue } \\
\text { medication }\end{array}$ \\
\hline SGRQ total score & $0.59 * * *$ & $0.56^{* * *}$ & $0.64^{* * * *}$ & $0.33^{* * *}$ \\
SGRQ symptoms score & $0.67^{* * *}$ & $0.58^{* * *}$ & $0.52^{* * *}$ & $0.33^{* * *}$ \\
SGRQ impact score & $0.54^{* * *}$ & $0.52^{* * *}$ & $0.60^{* * *}$ & $0.32^{* * *}$ \\
E-RS total score & $0.83^{* * *}$ & $0.80^{* * *}$ & $0.73^{* * *}$ & $0.35^{* * *}$ \\
FEV (trough) $^{\mathrm{b}}$ & -0.04 & -0.10 & $-0.13^{* *}$ & -0.13 \\
\hline
\end{tabular}

Notes: aSpearman rank order correlation coefficients: ${ }^{* * *} p<0.000 \mathrm{I} ;{ }^{* *} p<0.00 \mathrm{I}$. ${ }^{\circ}$ Morning pre-dose value. 'Single item measuring overall early morning COPD symptom severity. ${ }^{\mathrm{d}}$ Average score of 6 symptoms (cough, wheezing, shortness of breath, tightness in your chest, chest congestion, and difficulty bringing up phlegm).

Abbreviations: EMS, early morning symptoms; EMSCI, Early Morning Symptoms of COPD Instrument; E-RS, Evaluating Respiratory Symptoms; FEV ${ }_{1}$, forced expiratory volume in I second; SGRQ, St George's Respiratory Questionnaire. 


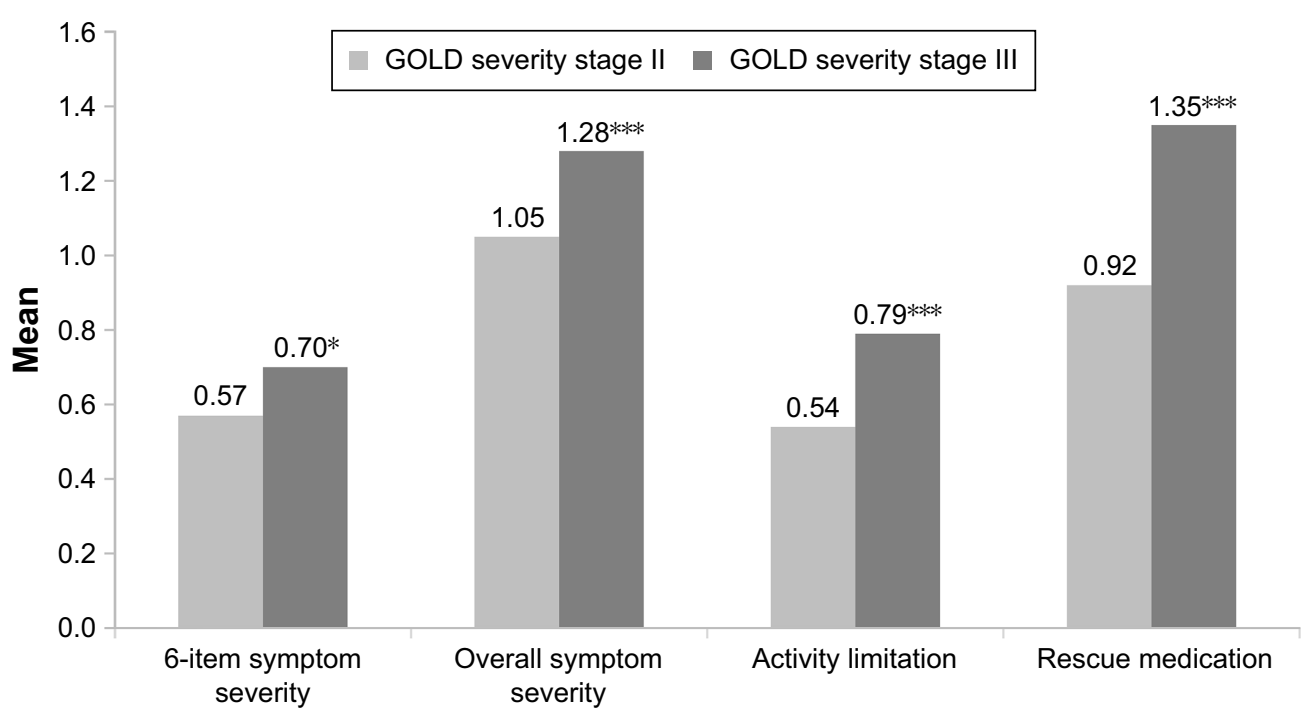

Figure $3 \mathrm{EMSCl}$ domain scores by GOLD severity stages II and III at baseline week.

Note: Significance level for comparison $* * * p<0.0001 ;{ }^{*} p<0.05$.

Abbreviations: EMSCI, Early Morning Symptoms of COPD Instrument; GOLD, Global initiative for chronic Obstructive Lung Disease.

the measure is able to distinguish between groups known to differ clinically (GOLD stages II and III, and between median-splits of SGRQ, and E-RS total scores).

The validity assessments in the current study suggest that either the 6-item symptom severity score or the overall symptom severity score could be used as an assessment of COPD EMS presence or severity. The 6-item score is based on more items than the overall score; it is therefore likely to be more precise because it combines scores on a range of symptoms, whereas the overall score does not take into account that some symptoms might be more bothersome than others. However, the overall symptom severity score may provide a simple single item measure for evaluating symptom-free days, which may be an important endpoint for trials evaluating mild COPD. The American Thoracic Society has provided a statement that an overall symptom measure is more appropriate than individual symptom items for evaluating symptom-free days. Whenever possible,

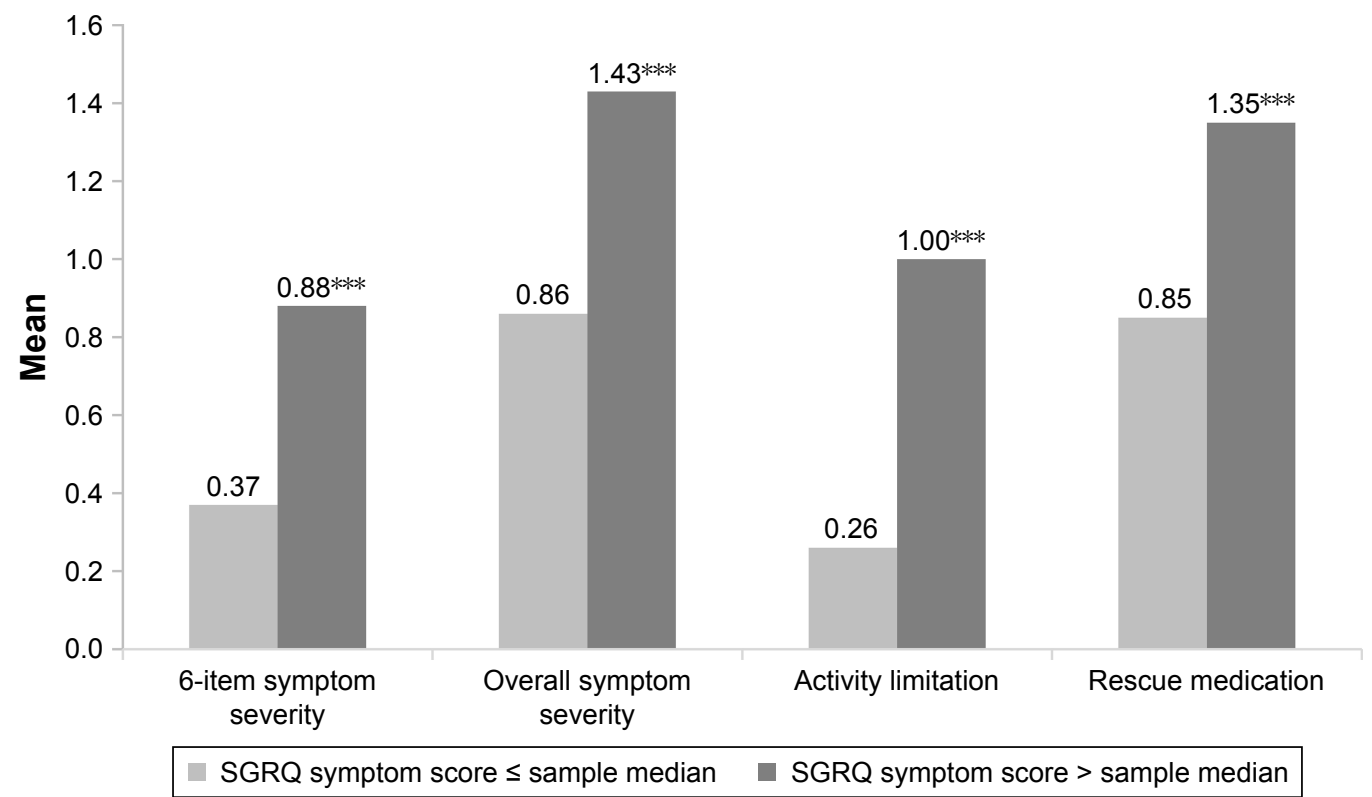

Figure $4 \mathrm{EMSCl}$ domain scores by SGRQ total score at baseline week.

Note: Significance level for comparison ${ }^{* * *} p<0.000$ I.

Abbreviations: EMSCI, Early Morning Symptoms of COPD Instrument; SGRQ, St George's Respiratory Questionnaire. 


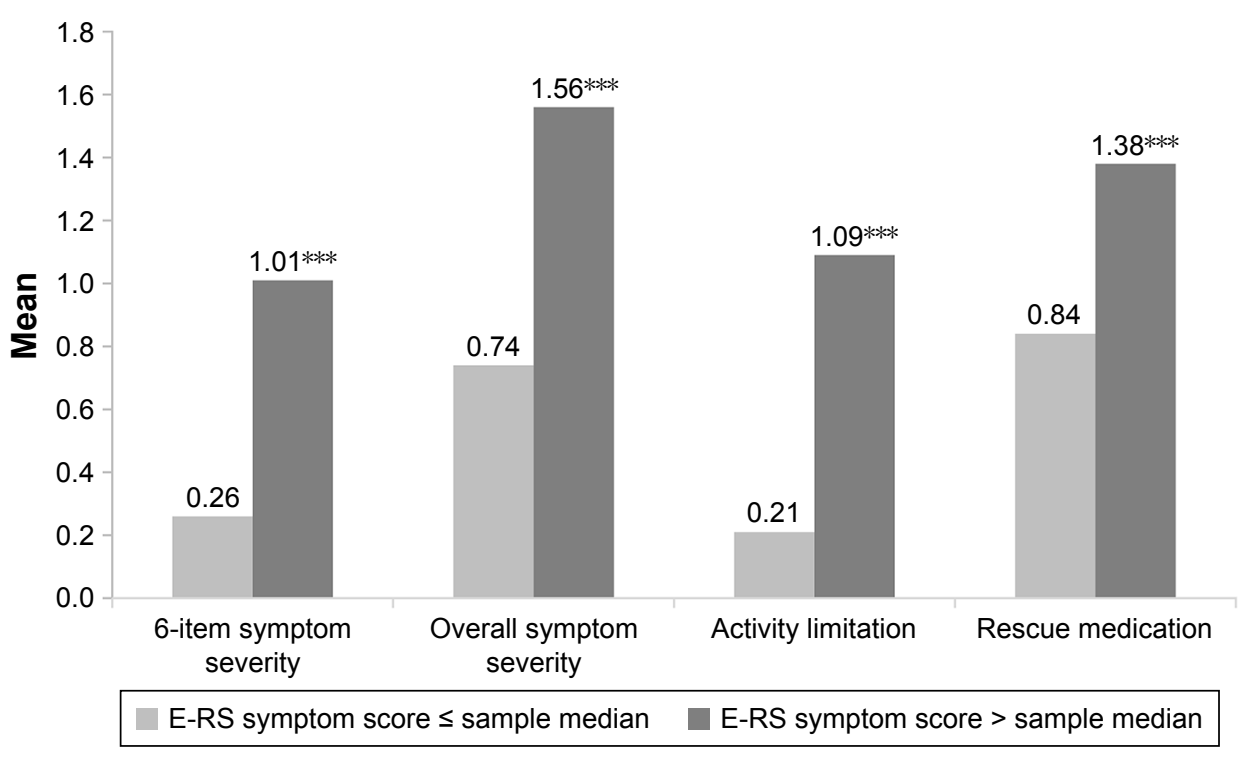

Figure $5 \mathrm{EMSCl}$ domain scores by E-RS scores at baseline week.

Note: Significance level for comparison ${ }^{* * *} p<0.0001$.

Abbreviations: EMSCI, Early Morning Symptoms of COPD Instrument; E-RS, Evaluating Respiratory Symptoms.

we recommend using both scores, as together they would provide the most comprehensive picture of EMS in patients with COPD. However, there may be cases where participant burden is a concern and a single item measure could be used to reduce this burden. The overall severity item has proven to be robust; however, it was evaluated in the context of its application alongside the 6 individual symptom severity questions and therefore responses may have been influenced by the responses given to the individual items. Further testing of this single item on its own is required to confirm these findings.

While there are other instruments that have been developed to examine EMS in patients with COPD, they have limitations that have been addressed with the EMSCI. For example, both the CDLM and the GCSQ were developed using a small sample of individuals. ${ }^{5}$ The EMSCI was developed with patient input to the point of saturation, providing evidence of content validity. ${ }^{11}$ The COPD-MSD and MEMSI also assess EMS of COPD; however, the former has not been validated, and the latter asks patients to recall their symptoms over a 1-week period. The results of this study demonstrate the reliability and validity of the EMSCI. Additionally, the EMSCI is designed to collect data every day and is therefore able to gather information about symptoms and limitations that typically vary on a day-to-day basis in COPD patients. Additionally, a daily recall period decreases the likelihood of recall bias.

As a daily diary, EMSCI can be used to evaluate outcomes that are relevant to COPD patients and reflect the patients' perspective: average severity as well as variability of COPD patients' experiences over time, as well as calculate symptom-free time periods. A related instrument to the EMSCI is the Nighttime Symptoms of COPD Instrument. ${ }^{25}$ It assesses nighttime symptoms of COPD and has been validated. ${ }^{26}$ The 2 measures were developed at the same time and have been used alongside one another in clinical trials and observational studies (such as NCT01462942, NCT01462929, and NCT03031769). These instruments would be suitable for use in a clinical trial. While the EMSCI could also be used in clinical practice to monitor patient outcomes, this has not been tested.

\section{Study limitations and future research}

A limitation of this study is the somewhat homogenous population. The patient population taken from the clinical trial consisted mainly of patients with COPD classified in GOLD stage II or III. Sample sizes of patients in higher (IV) or lower stages (I) were not large enough to be analyzed. Additionally, the study population was mostly White and did not provide a representative sample of patients with COPD. In this study, there was a weak relationship between $\mathrm{FEV}_{1}$ and symptoms. Although this is in line with previous research, ${ }^{27}$ it is possible that at higher levels of severity, this relationship could differ. However, it was not possible to test this with this sample size. Finally, this study used scores from the SGRQ and E-RS to examine convergent validity of the EMSCI. Other known measures which look at symptoms throughout the day in COPD were not used during the trial. Future research should examine the relationship between EMSCI scores and 
other known measures of COPD symptoms (for example, the Medical Research Council Dyspnea Scale). ${ }^{28}$

\section{Conclusion}

The EMSCI is a reliable and valid instrument to evaluate early morning COPD symptoms and activity limitation, and shows good internal consistency and test-retest reliability. Its robust psychometric properties suggest that it can be used for the evaluation of COPD patients' experience of EMS and as an outcome measure for evaluating the benefit of interventions. Further research is being conducted to determine definitions of clinically meaningful change to enable the interpretation of change scores on the EMSCI when evaluating interventions to reduce EMS.

\section{Acknowledgments}

The authors would like to thank the AUGMENT COPD study investigators and also Michelle Mocarski. Michelle was an employee of Forest Research Institute and contributed to the psychometric evaluation of the measure. This study was supported by Forest Research Institute, a wholly-owned subsidiary of Forest Laboratories Inc., New York, NY, USA, and by Almirall S.A., Barcelona, Spain. Evidera was paid by Forest Research Institute for the analyses and reporting. Medical writing support was provided by Hayley Syrad and Natalia Hawken, employees of Evidera. Evidera was paid by AstraZeneca to provide medical writing support.

\section{Disclosure}

E Garcia Gil was an employee at Almirall S.A. during the analyses and was involved in this study, and is currently an employee and stockholder at AstraZeneca. E Zaiser and A Hareendran, who were involved in conceptualizing the analyses plan and interpreting results, are employees of Evidera. The authors report no other conflicts of interest in this work.

\section{References}

1. Global Initiative for Chronic Obstructive Lung Disease (GOLD). Global Strategy for the Diagnosis, Management and Prevention of COPD; 2016. Available from: http://goldcopd.org/. Accessed January 12, 2017.

2. Vogelmeier C, Partridge M, Miravitlles M, et al. Perception of symptom variability in patients with severe COPD: impact on morning activities and therapeutic behavior. Poster presented at: American Thoracic Society Conference 2009; San Diego, CA, USA.

3. Kessler R, Partridge MR, Miravitlles M, et al. Symptom variability in patients with severe COPD: a pan-European cross-sectional study. Eur Respir J. 2011;37(2):264-272.

4. Lopez-Campos JL, Calero C, Quintana-Gallego E. Symptom variability in COPD: a narrative review. Int J Chron Obstruct Pulmon Dis. 2013;8: 231-238.
5. Partridge MR, Schuermann W, Beckman O, Persson T, Polanowski T. Effect on lung function and morning activities of budesonide/formoterol versus salmeterol/fluticasone in patients with COPD. Ther Adv Respir Dis. 2009;3(4):1-11.

6. Roche N, Chavannes NH, Miravitlles M. COPD symptoms in the morning: impact, evaluation and management. Respir Res. 2013;14:112.

7. Patrick DL, Burke LB, Gwaltney CJ, et al. Content validity-establishing and reporting the evidence in newly developed patient-reported outcomes (PRO) instruments for medical product evaluation: ISPOR PRO good research practices task force report: part 1-eliciting concepts for a new PRO instrument. Value Health. 2011;14(8):967-977.

8. Partridge MR, Miravitlles M, Stahl E, Karlsson N, Svensson K, Welte T. Development and validation of the capacity of daily living during the morning questionnaire and the global chest symptoms questionnaire in COPD. Eur Respir J. 2010;36(1):96-104.

9. Globe G, Currie B, Leidy NK, et al. Development of the chronic obstructive pulmonary disease morning symptom diary (COPD-MSD). Health Qual Life Outcomes. 2016;14(1):104.

10. Garrow AP, Khan N, Tyson S, Vestbo J, Singh D, Yorke J. The development and first validation of the Manchester Early Morning Symptoms Index (MEMSI) for patients with COPD. Thorax. 2015; 70(8):757-763.

11. Palsgrove A, Houghton K, Hareendran A, et al. PRS51 the development of the early morning symptoms of COPD instrument (EMSCI). Value Health. 2011;14(7):A496-A497.

12. US Department of Health Human Services FDA Center for Drug Evaluation Research; US Department of Health Human Services FDA Center for Biologics Evaluation Research; US Department of Health and Human Services FDA Center for Devices and Radiological Health. Guidance for industry: patient-reported outcome measures: use in medical product development to support labeling claims: draft guidance. Health Qual Life Outcomes. 2006;4:79.

13. European Medicines Agency, Committee for Medicinal Products for Human Use (CHMP), (EWP) EWP. Reflection paper on the regulatory guidance for the use of health-related quality of life (HRQL) measures in the evaluation of medicinal products; July 2005. Available from: http://www.emea.europa.eu/docs/en_GB/document_library/Scientific_guideline/2009/09/WC500003637.pdf. Accessed October 23, 2012.

14. Streiner DL, Norman GR. Health Measurement Scales: A Practical Guide to Their Development and Use. 4th ed. New York, NY: Oxford University Press; 2008.

15. Mahler DA, O’Donnell DE. Dyspnea: Mechanisms, Measurement, and Management. 3rd ed. New York, NY: CRC Press; 2014.

16. ClinicalTrials.gov. Efficacy, Safety and Tolerability of Aclidinium Bromide/Formoterol Fumarate Compared With Formoterol Fumarate in Patients With Moderate to Severe Chronic Obstructive Pulmonary Disease (COPD); 2016. Available from: https://clinicaltrials.gov/ct2/ show/NCT01437397. Accessed January 12, 2017.

17. Leidy NK, Wilcox TK, Jones PW, et al; EXACT-PRO Study Group. Development of the EXAcerbations of chronic obstructive pulmonary disease tool (EXACT): a patient-reported outcome (PRO) measure. Value Health. 2010;13(8):965-975.

18. Jones PW, Quirk FH, Baveystock CM. The St George's Respiratory Questionnaire. Respir Med. 1991;85 (Suppl B):25-31; discussion 3-7.

19. Hurst H, Bolton J. Assessing the clinical significance of change scores recorded on subjective outcome measures. J Manipulative Physiol Ther. 2004;27(1):26-35.

20. Junghard O, Lauritsen K, Talley NJ, Wiklund IK. Validation of seven graded diary cards for severity of dyspeptic symptoms in patients with non ulcer dyspepsia. Eur J Surg Suppl. 1998(583):106-111.

21. Leidy NK, Revicki DA, Geneste B. Recommendations for evaluating the validity of quality of life claims for labeling and promotion. Value Health. 1999;2(2):113-127.

22. Nunnally JC, Bernstein IH. Psychometric Theory. 3rd ed. New York, NY: McGraw-Hill; 1994. 
23. Cohen J. Statistical Power Analysis for the Behavioral Sciences. 2nd ed. Hillsdale, NJ: Lawrence Erlbaum Associates; 1988.

24. Jones PW, Brusselle G, Dal Negro RW, et al. Patient-centred assessment of COPD in primary care: experience from a cross-sectional study of health-related quality of life in Europe. Prim Care Respir J. 2012;21(3):329-336.

25. Hareendran A, Palsgrove AC, Mocarski M, et al. The development of a patient-reported outcome measure for assessing nighttime symptoms of chronic obstructive pulmonary disease. Health Qual Life Outcomes. 2013;11:104.
26. Mocarski M, Zaiser E, Trundell D, Make BJ, Hareendran A. Evaluation of the psychometric properties of the nighttime symptoms of COPD instrument. Int J Chron Obstruct Pulmon Dis. 2015;10:475-487.

27. Nishimura K, Izumi T, Tsukino M, Oga T. Dyspnea is a better predictor of 5-year survival than airway obstruction in patients with COPD. Chest. 2002;121(5):1434-1440.

28. Bestall JC, Paul EA, Garrod R, Garnham R, Jones PW, Wedzicha JA. Usefulness of the Medical Research Council (MRC) dyspnoea scale as a measure of disability in patients with chronic obstructive pulmonary disease. Thorax. 1999;54(7):581-586. 


\section{Supplementary material}

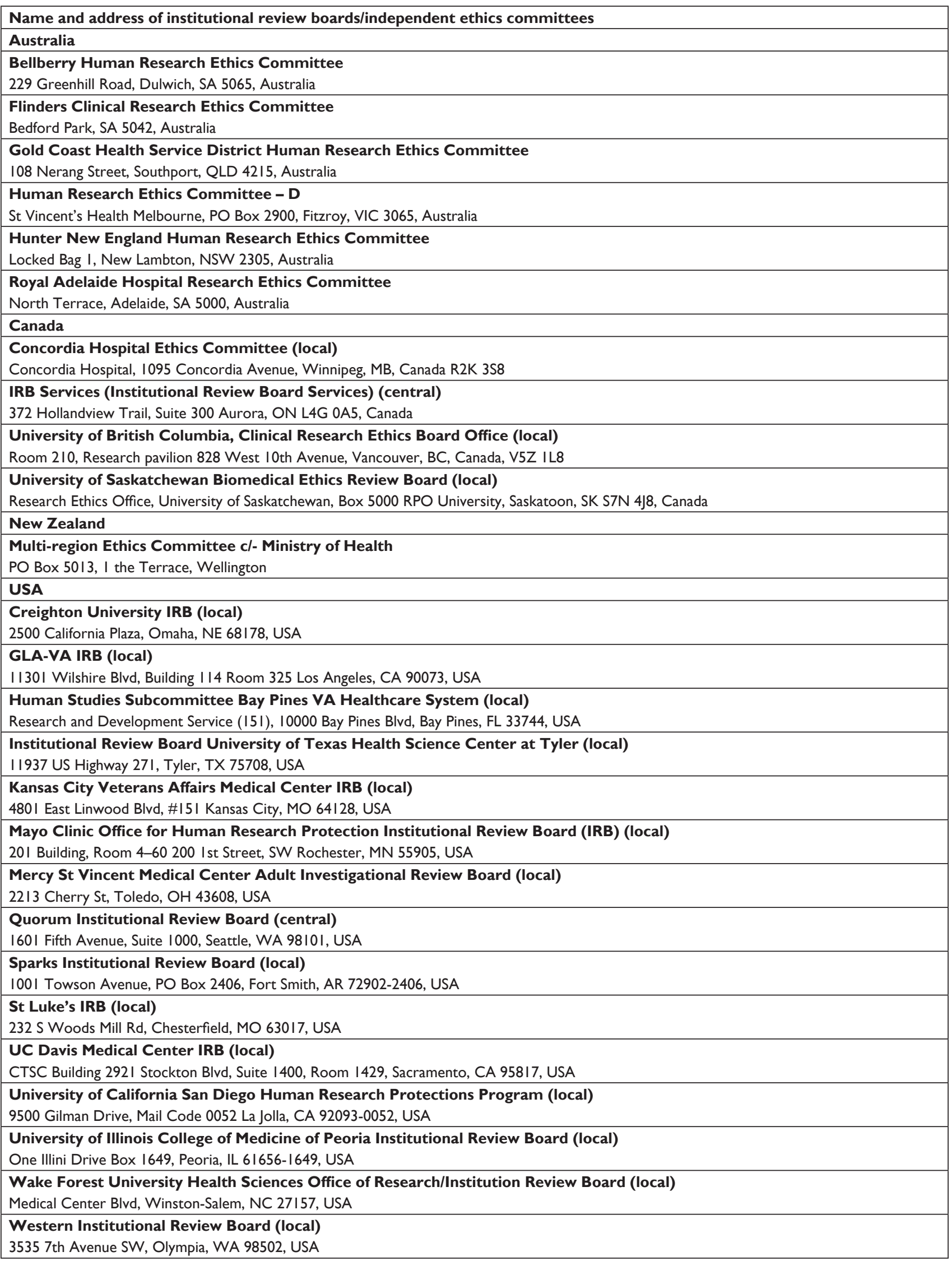


International Journal of COPD

\section{Publish your work in this journal}

The International Journal of COPD is an international, peer-reviewed journal of therapeutics and pharmacology focusing on concise rapid reporting of clinical studies and reviews in COPD. Special focus is given to the pathophysiological processes underlying the disease, intervention programs, patient focused education, and self management protocols.

This journal is indexed on PubMed Central, MedLine and CAS. The manuscript management system is completely online and includes a very quick and fair peer-review system, which is all easy to use. Visit http://www.dovepress.com/testimonials.php to read real quotes from published authors 